\title{
SIGNIFICADO DA OCORRÊNCIA DE FOSFATOS E BORATOS DE ALUMÍNIO NO CONTATO ENTRE OS SUPERGRUPOS RIO PARAÚNA E ESPINHAÇO NA REGIÃO DE DIAMANTINA, MINAS GERAIS, BRASIL
}

\author{
Adolf Heinrich Horn (*), Giulio Morteani (**) \& Dietrich Ackermand (***)
}

\begin{abstract}
Within a muscovite-kyanite quartzite and/or muscovite-kyanite schist at the contact between the Upper Archean Barão de Guacuí Formation and the Bandeirinha Formation near Diamantina (State of Minas Gerais, Brazil) five rock types defining mineral assemblages can be found: 1: lazulite, kyanite, 2: lazulite, augelite, kyanite; 3: lazulite, amblygonite, tourmaline; 4: lazulite, svanbergite, tourmaline, and 5: dumortierite, kyanite. Additional phases in all rock types are quartz and muscovite/sericite. The whole rock chemistry influences the chemical composition of the individual minerals.

These ocurrences are interpreted as precipitation of aluminium, phosphorous and boron-rich sedimentary rocks in highly alcaline lakes under sabka conditions in the Middle Proterozoic on the Archean metasediments of the Baraõ de Guacuí Formation.

During the Brasiliano event (680-450Ma) a greenschist metamorphism and associated tectonism with thrusting the actual texture and foliation were formed. Mobilization of lazulite and its deposition into quartz veins, cutting the over- and underlain units occurred, too.

The aluminophosphate minerals get importance because they could help to define more precisely the contact between the Rio Paraúna and Espinhaço supergroups.
\end{abstract}

\section{INTRODUÇ̃̃O}

Excluindo-se a ampla literatura sobre a apatita, existem poucos trabalhos sobre as ocorrências de minerais de fosfato em rochas metamórficas. Trabalhos sobre a estabilidade $\mathrm{P}-\mathrm{T}$ de fosfatos de $\mathrm{Al}$ são escassos como também sobre o significado destas ocorrências no âmbito da geologia regional.

No Brasil, as rochas que contêm lazulita são mencionadas na literatura sobre a Bahia (Bank 1972; Cassedanne et al. 1989; Cassedanne 1990) e de Minas Gerais (Eberle 1970, 1972; Cassedanne \& Cassedanne 1975; Hoffmann 1979), mas descrições detalhadas a respeito da petrologia e química ainda não são suficientes.

Neste artigo, queremos apresentar uma descrição petrológica resumida incluindo a composição mineralógica, as relações texturais quer de campo, quer de lâmina, a relação com a composição química dos muscovita-cianita-quartzitos e muscovita-cianitaquartzo-xistos que contêm lazulita, svanbergita, augelita, ambligonita, dumortierita e turmalina e as informações que se obtém da sua ocorrência em relação para o desenvolvimento do Rift Espinhaço, especialmente para o contato entre os supergrupos Rio Paraúna e Espinhaço.

Estas rochas foram até o presente momento incluídas na parte superior da Formação Barão de Guacuí, topo do Supergrupo Rio Paraúna, na região de Diamantina.
Alguns aspectos e observações parecem poder colocar estas rochas na base do Supergrupo Espinhaço e especialmente da Formação Bandeirinha.

As rochas estudadas neste artigo são um dos raros exemplos de fosfatos sedimentares de idade PréCambriana e permitem, talvez uma melhor avaliação das condições desta região durante a fase madura do Rift Espinhaço. A figura 1 mostra as relações de campo e os afloramentos amostrados.

\section{LITOESTRATIGRAFIA E PETROGRAFIA}

A seqüência litológica típica que se encontra na literatura baseia-se nas nomenclaturas utilizadas por Pflug \& Carvalho (1964), Pflug (1965, 1968), Dorr (1969), Schöll \& Fogaça (1979, 1981), Pflug et al. (1980), Fogaça \& Schöll (1984), Herrgesell \& Pflug (1986), Machado et al. (1989), Almeida Abreu et al. (1992), Knauer (1993), Almeida Abreu (1989, 1994), Dussin (1994) e Silva (1995).

A seqüência começa com o Complexo Basal de idade Arqueana, formado por granitos, granitóides, migmatitos, para- e ortognaisses. Na área estudada ele é representado pelo Granito Gouveia, de idade Arqueana (+2,8Ga; Almeida Abreu 1993, Silva 1995; Dussin 1994).

A seguir, discordantemente, foi depositado o Supergrupo Paraúna, formado pelo Grupo Pedro Pereira, inexistente na região da investigação, e o Grupo 


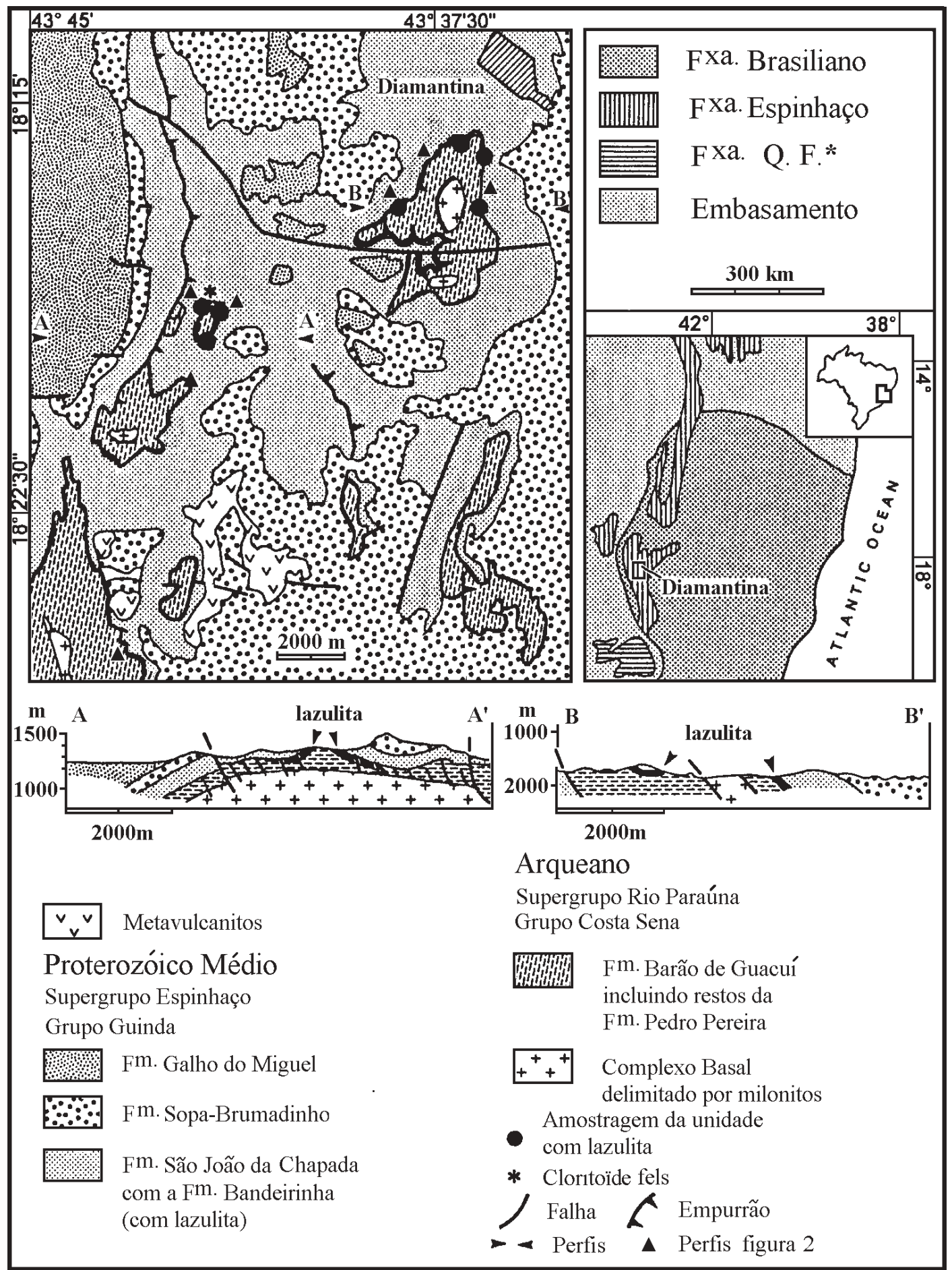

Figura 1: Mapa simplificado da região de Diamantina (segundo Schöll \& Fogaça 1981, modificado). Estão indicados os pontos de amostragem e ocorrências de lazulita. * Quadrilátero Ferrífero

Figure 1: Sketch map of the Diamantina region (after Schöll \& Fogaça 1981, modified). The sampling points and the limite occurrences are indicated.

Costa Sena. Estes grupos são considerados pela maioria dos autores como restos de um Complexo Greenstone (Pflug 1965, 1968; Dorr 1969, Dussin 1994), com posicionamento igual ao Supergrupo Rio das Velhas. Outros autores consideram as partes superiores do Grupo Costa Sena, isto é, a Formação Bandeirinha, como uma seqüência depositada discordante sobre o Supergrupo Rio Paraúna (Almeida Abreu 1993; Silva
1995) no início do Rift Espinhaço. Datações em rochas intrusivas básicas e efusivas ácidas na base desta unidade mostram idades entre 1,2 a 1,6Ga (Almeida Abreu 1993, Silva 1995; Dussin 1994; U-Pb em zircão).

O Grupo Costa Sena, antigamente dividido em Formação Barão de Guacuí e Formação Bandeirinha (Schöll \& Fogaça 1979, 1981; Pflug 1968, Dussin 1994) contém hoje, para vários autores, somente a Formação 
Barão de Guacuí. A Formação Bandeirinha foi colocada na base do Supergrupo Espinhaço (Almeida Abreu 1993, Silva 1995; Dussin 1994).
De acordo com Almeida Abreu (1994) o protólito da Formação Barão de Guacuí é uma seqüência vulcano-sedimentar transicional para rochas

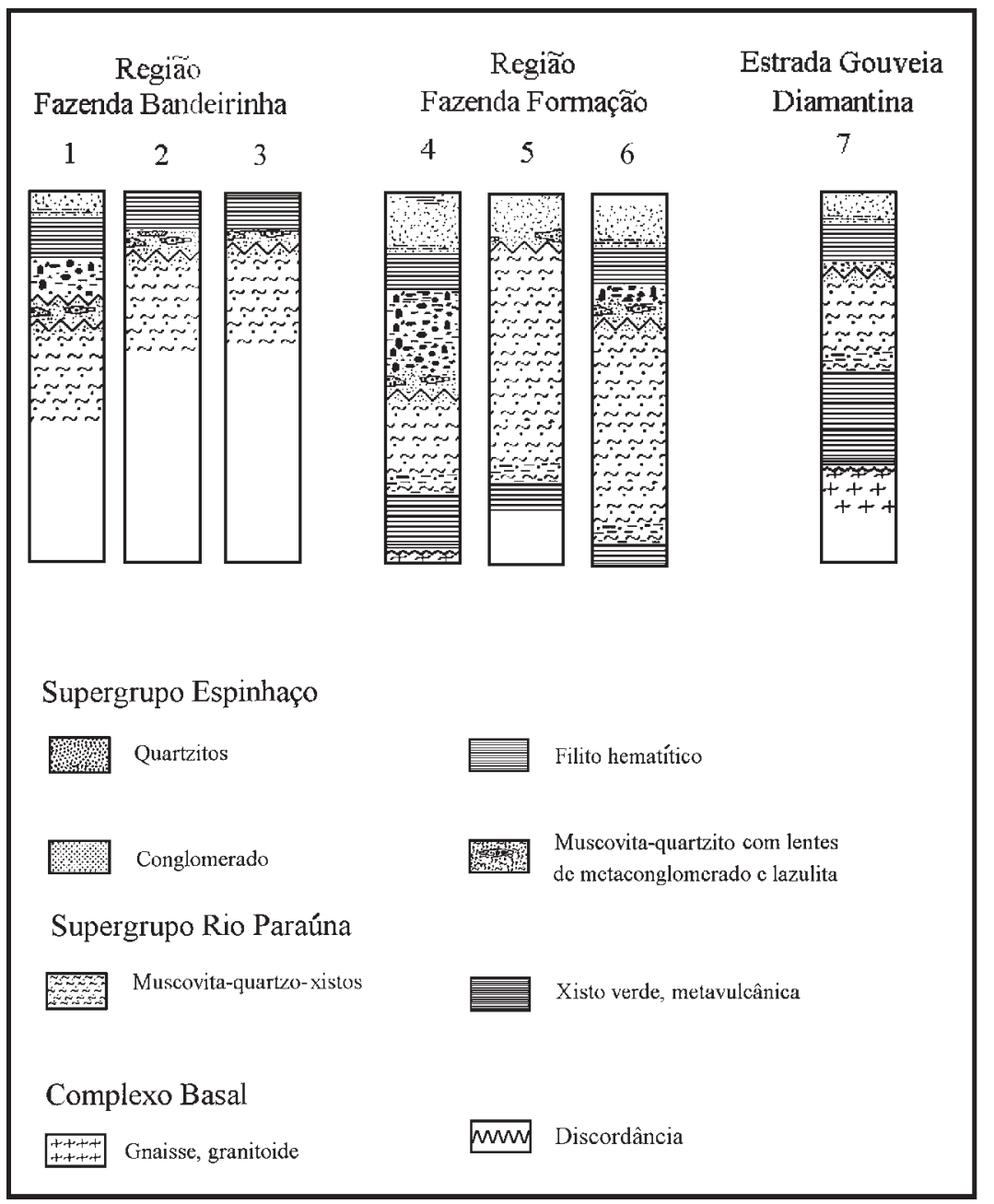

Figura 2: Comparação esquematizada de colunas estratigráficas de várias regiões da área de trabalho. Observações de Campo. A localização dos perfis é indicada na figura 1.

Figure 2: Schematic comparison of stratigraphic columns of diffferent regions of the working area. The localization of the profiles is indicated in figure 1.

Investigando à localidade na rodovia, logo após a saida de Gouveia, em direção a Diamantina (figura 2, perfil 7), a Formação Barão de Guacuí foi subdividida, neste trabalho, devido a distribuição de rochas ígneas e sedimentares, em duas unidades distintas, uma inferior e outra superior.

A unidade inferior é formada principalmente por rochas metavulcânicas como xisto verde, talco-quartzoxistos e subordinadamente muscovita-quartzo-xistos e muscovita-quartzitos com lentes de quartzo-pórfiro. Normalmente não ocorre cianita.

A unidade superior é constituída por cianitamuscovita-quartzitos, cianita-muscovita-quartzo-xistos e bandas de quartzito extremamente deformados, dobrados e boudinados. Em torno dos boudins de quartzo ocorrem agregados de cristais de cianita de cor branca a levemente verde de até $15 \mathrm{~cm}$ de largura. Esta subdivisão foi observada também em outras localidades da área de trabalho (figura 2, perfis 4 a 6). sedimentares. Outros autores como Silva (1995), acreditam que uma parte é formada por milonitos do embasamento. Knauer (1989) acredita que uma parte é de material do Supergrupo Espinhaço cisalhado. Knauer \& Schrank (1994) mencionam a existência de um paleossolo na parte superior da Formação Rio Paraúna baseando-se, entre outros argumentos citados, no seu alto teor de alumínio.

Acima do Supergrupo Rio Paraúna, com deposição discordante, segue primeiro o Supergrupo Espinhaço, com o grupo Guinda (Almeida Abreu, 1994), por alguns autores ainda denominado Diamantina (Dussin, 1994), o Grupo Conselheiro Mata e depois o Supergrupo São Francisco (Figura 2).

Foi visitado o contato entre os dois supergrupos em vários locais na região de Diamantina (figura 1) como a leste da Serra da Miúda, na região da Fazenda Bandeirinha, na janela estrutural da Fazenda Formação e ao norte da cidade Gouveia. A figura 1 mostra o mapa 
geológico e a figura 2, perfis esquemáticos desta região.

Nos perfis (figura 2) que mostram as relações do contato entre os supergrupos Rio Paraúna e Espinhaço observa-se uma variação entre as rochas com minerais de fosfato de alumínio e com minerais de borato de alumínio. As rochas que não contém estes minerais possuem duas foliações, das quais uma poderia ser atribuída ao Evento Deformational Espinhaço e a outra ao Evento Brasiliano. Esta rocha tem sempre grandes quantidades de cianita e uma estrutura relativamente homogênea de um muscovita-quartzo-xisto. É típica a ocorrência, no contato com a rocha encaixante, de grande quantidade de veios de quartzo contendo cristais de cianita até $10 \mathrm{~cm}$ de comprimento.

Nas rochas com ocorrência de lazulita foi observado somente uma foliação, pequenos cristais de cianita e lentes de espessura variada $(<1 \mathrm{~m}$ até $5 \mathrm{~m})$ de conjuntos de grãos de quartzo, às vezes recristalizadas (ribbon quartz), às vezes de morfologia arredondada e irregular, que parecem com conglomerados de grãos e seixos de quartzo. Estes lentes tem sempre orientação +paralela ao contato.

\section{MINERALOGIA}

Quartzo forma agregados policristalinos de grãos alongados, mas também ocorre como uma matriz de granulação fina. Os boudins de quartzo de granulação grosseira e forma arredondada, dão aparência macroscópica de um metaconglomerado tectonizado. Estes boudins, às vezes, têm largura de até $15 \mathrm{~cm}$ e um comprimento de até $5 \mathrm{~m}$. Muitas vezes, os contatos dos grãos poligonais indicam anelamento pósdeformacional com recristalização tardia.

Muscovita existe em, no mínimo, duas gerações. A mais antiga forma plaquetas orientadas responsáveis pela xistosidade proeminente e a aparência soft da rocha. Esta mica branca muitas vezes está intimamente intercrescida com grandes cristais de cianita corroidos. Uma análise típica é anotada na tabela 1 .

A geração mais nova é de uma granulometria muito fina (sericita), e muitas vezes intercrescida simplectiticamente com lazulita e svenbergita.

Cianita está presente em duas gerações distintas em concentração até 20 vol\% nos tipos de rocha 1,2 e 5 . A geração mais antiga é principalmente formado por grãos euédricos a subédricos.

A geração mais nova (secundária), incluindo muitas vezes a geração primária da lazulita, é anédrica a poiquiloblástica por causa das pequenas inclusões de lazulita. A composição normal é mostrada na tabela 1.

Turmalina é um constituinte muito comum nas rochas estudadas. Normalmente é subédrica a euédrica e mostra um zoneamento com zonas anédricas de cor verde escuro e zonas euédricas de cor verde-pálido. Às vezes forma agregados do tipo garben e/ou em forma de sóis. Uma análise típica para a variedade verdeescuro é mostrada na tabela 1 .

Ilmenohematita/hematita está presente em todas as rochas estudadas. Análises preliminares com a microssonda mostram uma variação considerável na relação $\mathrm{Fe} / \mathrm{Ti}$.

Talco ou pirofilita foram identificados por pequenos DRX-peaks em duas amostras.

Lazulita, em amostras-de-mão possui uma coloração azul muito pálida é de granulação fina e normalmente disseminada. Cristais individuais ou agregados com a sua cor azul-escuro típica são muito raros. Encontra-se também em veios de quartzo secundários onde forma cristais típicos, os quais possuem qualidade gemológica.

As investigações microscópicas mostraram que a lazulita ocorre na rocha do tipo 1 como um material policristalino, azul-pálido, granulometria fina e com intercrescimento simplectítico com os cristais grandes de cianita.

Nas paragêneses do tipo 2 a lazulita ocorre principalmente, em manchas de lazulita-muscovitasimplectito.

No tipo de rocha 3 a lazulita aparece como grandes grãos anédricos, azul-escuro e com muitas inclusões de muscovita, quartzo, ambligonita e turmalina euédrica.

Nas amostras do tipo 4 a lazulita tem muitas vezes margens irregulares de svanbergita.

A composição dos diferentes tipos são visíveis na tabela 1. Observa-se uma variação nos teores de $\mathrm{Fe}^{++}$ (diminui) e $\mathrm{Mg}^{++}$(aumenta) do tipo 1 para 4 .

Augelita encontra-se em grãos anédricos, de cor marrom-clara a transparente com refração baixa e cor de interferência amarela. Muitas vezes ela ocorre em torno de grãos de lazulita. O teor é de até $10 \%$ da rocha do tipo 2. A composição é mostrada na tabela 1 .

Ambligonita foi encontrada somente no tipo 3. Ela pode ser encontrada em grãos prismáticos euédricos, em pequenos veios ou em quartzo mobilizado e como grãos anédricos junto a cianita e lazulita. Às vezes os grãos mostram uma fase marrom que não pode ser separada ou determinada por microscópio ou microssonda. A composição da ambligonita é mostrada na tabela 1 .

Svanbergita forma manchas irregulares na matriz rica em quartzo e mica, onde está intercrescida intimadamente com a lazulita, mas pode formar também grãos. Foi encontrada somente no tipo 4, caracterizado por elevadíssimos teores de $\mathrm{SrO}$ (4296ppm). A composição da svanbergita é mostrada na tabela 1 .

Dumortierita foi encontrada por outros pesquisadores (Schöll \& Fogaça 1981) somente em agregados de agulhas junto à muscovita e quartzo das rochas do tipo 5. Este tipo é caracterizado pela falta de fosfatos e conseqüentemente, baixos teores em $\mathrm{P}_{2} \mathrm{O}_{5}$ na análise de rocha total. Durante este trabalho encontrou-se agulhas de dumortierita também em rochas do tipo 1 (Fazenda Bandeirinha) onde o teor de 2 é muito elevado (até 2670ppm). Estas agulhas foram encontradas em planos de falhas de empurrão N-S mergulhando para leste. A composição da dumortierita é mostrada na tabela 1 . 


\begin{tabular}{|c|c|c|c|c|c|c|c|c|c|c|c|}
\hline Mineral & Lazul. & Lazul. & Lazul. & Lazul. & Augel & Ambli & Svan & Musc & Tour & Cyan. & Dumo \\
\hline Num. r. & 1 & 2 & 3 & 4 & 2 & 3 & 4 & 1 & 3 & 5 & 5 \\
\hline Num. am. & 5726 & 5711 & 5717 & 5722 & 5711 & 5717 & 5716 & 5726 & 5717 & 5725 & 5725 \\
\hline $\mathrm{SiO}_{2}$ & 0.22 & 0.66 & 0.64 & 0.08 & 0.25 & 0.04 & 0.36 & 50.53 & 36.73 & 37.31 & 31.03 \\
\hline $\mathrm{TiO}_{2}$ & -- & -- & -- & 0.08 & 0.03 & -- & 0.43 & -- & 0.19 & 0.02 & 0.80 \\
\hline $\mathrm{Al}_{2} \mathrm{O}_{3}$ & 33.15 & 32.99 & 31.39 & 31.10 & 46.91 & 34.95 & 33.05 & 38.00 & 32.28 & 61.29 & 60.04 \\
\hline $\mathrm{Fe}_{2} \mathrm{O}_{3}{ }^{*}$ & -- & -- & -- & -- & 1.50 & 1.21 & -- & -- & -- & 0.47 & 0.39 \\
\hline $\mathrm{FeO}^{*}$ & .47 & 3.51 & 4.76 & 7.97 & -- & -- & 0.11 & 1.28 & 12.26 & -- & -- \\
\hline $\mathrm{MgO}$ & 13.94 & 11.47 & 11.35 & 9.22 & 0.07 & -- & 0.02 & 0.09 & 3.07 & -- & 0.58 \\
\hline $\mathrm{CaO}$ & -- & 0.02 & 0.01 & -- & 0.01 & -- & 1.61 & -- & 0.02 & 0.01 & -- \\
\hline $\mathrm{Na}_{2} \mathrm{O}$ & -- & -- & -- & -- & -- & 0.01 & 0.01 & 0.20 & 1.2 & 0.02 & -- \\
\hline $\mathrm{K}_{2} \mathrm{O}$ & 0.01 & 0.1 & -- & 0.02 & 0.03 & 0.01 & 0.14 & 7.54 & 0.05 & 0.02 & -- \\
\hline $\mathrm{P}_{2} \mathrm{O}_{5}$ & 49.46 & 47.74 & 46.08 & 46.74 & 36.40 & 51.27 & 26.35 & 0.20 & 0.04 & 0.04 & 0.04 \\
\hline $\mathrm{SO}_{3}$ & 0.02 & 0.02 & -- & -- & 0.05 & 0.01 & 7.79 & -- & -- & -- & 0.01 \\
\hline $\mathrm{As}_{2} \mathrm{O}_{3}$ & -- & 0.22 & 0.17 & -- & 0.29 & -- & -- & -- & -- & -- & --- \\
\hline $\mathrm{SrO}$ & 0.03 & -- & -- & -- & -- & -- & 17.98 & -- & -- & -- & $\overline{---}$ \\
\hline $\mathrm{La}_{2} \mathrm{O}_{3}$ & 0.08 & -- & -- & -- & -- & -- & 0.68 & 0.02 & -- & 0.03 & -- \\
\hline $\mathrm{Ce}_{2} \mathrm{O}_{3}$ & 0.00 & -- & -- & 0.05 & -- & -- & 1.36 & 0.01 & -- & 0.12 & --- \\
\hline $\mathrm{F}$ & 0.35 & 2.12 & 2.28 & 0.08 & 1.92 & 2.66 & 0.72 & -- & -- & 0.17 & --- \\
\hline Total & 97.58 & 97.96 & 95.72 & 95.31 & 86.65 & 89.04 & 90.31 & 97.86 & 85.98 & 99.43 & 92.91 \\
\hline $\mathrm{Si}$ & 0.01 & 0.03 & 0.03 & -- & 0.02 & -- & 0.03 & 6.36 & 7.24 & 2.03 & 5.99 \\
\hline Ox/.unid. & 9 & 9 & 9 & 9 & 11 & 8 & 11 & 22 & 29 & 10 & 33 \\
\hline $\mathrm{Ti}$ & -- & -- & -- & -- & -- & -- & 0.02 & -- & 0.03 & -- & 0.12 \\
\hline $\mathrm{Al}$ & 1.90 & 1.90 & 1.86 & 1.89 & 3.67 & 1.87 & 3.00 & 5.63 & 7.50 & 3.93 & 13.67 \\
\hline $\mathrm{Fe}^{3+}$ & -- & -- & -- & -- & 0.07 & 0.04 & -- & -- & -- & 0.02 & 0.06 \\
\hline $\mathrm{Fe}^{2+}$ & 0.02 & 0.14 & 0.20 & 0.34 & -- & -- & 0.01 & 0.14 & 2.02 & -- & --- \\
\hline $\mathrm{Mg}$ & 1.01 & 0.83 & 0.85 & 0.71 & 0.01 & -- & -- & 0.02 & 0.90 & -- & 0.17 \\
\hline $\mathrm{Ca}$ & -- & -- & -- & -- & -- & -- & 0.13 & -- & -- & -- & --- \\
\hline $\mathrm{Na}$ & -- & -- & -- & -- & -- & -- & -- & 0.05 & 0.46 & -- & --- \\
\hline $\mathrm{K}$ & -- & 0.01 & -- & -- & -- & -- & 0.01 & 1.21 & 0.01 & -- & --- \\
\hline$P$ & 2.03 & 1.97 & 1.96 & 2.04 & 2.05 & 2.27 & 1.72 & 0.02 & 0.01 & -- & 0.01 \\
\hline$S$ & -- & -- & -- & -- & -- & -- & 0.32 & -- & -- & -- & -- \\
\hline As & -- & 0.01 & 0.01 & -- & 0.01 & -- & -- & -- & -- & -- & --- \\
\hline $\mathrm{Sr}$ & -- & -- & -- & -- & -- & -- & 0.80 & -- & -- & -- & --- \\
\hline $\mathrm{La}$ & 0.02 & -- & -- & -- & -- & -- & 0.02 & -- & -- & -- & --- \\
\hline $\mathrm{Ce}$ & 0.04 & -- & -- & -- & -- & -- & 0.04 & -- & -- & -- & --- \\
\hline$F$ & 0.05 & 0.33 & 0.36 & 0.01 & 0.40 & 0.44 & 0.18 & -- & -- & 0.03 & --- \\
\hline
\end{tabular}

Tabela1: Análises representativos dos várias minerais típicos. (*) ferro total calculado como $\mathrm{Fe}^{2+}$ ou $\mathrm{Fe}^{3+}$ respectivamente; (**) válido também para os tipos de rocha 2 a 5; (***) também para o tipo 4.

$\mathrm{B}$ e $\mathrm{OH}^{-}$não foram analizados.

Table 1: Representative analises of the different tipic minerals. (*) total iron calculated as $\mathrm{Fe}^{2=}$ or $\mathrm{Fe}^{3+} ;\left({ }^{* *}\right)$ válido also for the rock types 2 to 5; (***) also for the type 4. $\mathrm{B}$ and $\mathrm{OH}^{-}$was not analysed. 


\section{MÉTODOS ANALÍTICOS}

A análise química total foi efetuada em 25 amostras. Elementos de terras raras (ETR), Ta, Sc, Zr, Hf, Cs, U e Th foram determinadas por ativação neutrônica (AN), Li com espectrometria de absorção atômica (EAA), FeO com colorimetria e B com espectrometria de emissão atômica com excitação de plasma inductiva acoplada (ICP-EEA). Os demais elementos foram dosados via fluorescência de raios $\mathrm{X}$ (FRX) com equipamento Siemens e pastilhas fundidas de Tetraborato de Li.

A microssonda que foi usada para a determinação da composição dos minerais é a Cameca Camebax Microbeam com quatro detectores de comprimento de onda dispersiva e o programa de correção on line PAP.

\section{COMPOSIÇÃO QUÍMICA TOTAL}

Através das paragêneses dos minerais e da composição química total, as rochas que contêm fosfatos podem ser divididas em cinco tipos (1 a 5) devido a assembléias típicas de minerais, mostrados e descritos na tabela 2:

Em todas rochas ocorrem quartzo e muscovita/ sericita.

1. Rocha com lazulita e cianita

2. Rocha com lazulita, svanbergita e cianita

3. Rocha com lazulita, ambligonita e turmalina

4. Rocha com lazulita, svanbergita e turmalina

5. Rocha com dumortierita e cianita

A composição total destas rochas ( tipos 1 a 5) é mostrada na tabela 2. É possível de ver que dos tipos 1 a 5 os teores de $\mathrm{P}_{2} \mathrm{O}_{5}$ e $\mathrm{Mg}^{++}$diminuirem claramente. Os teores de $\mathrm{Fe}^{++}$e $\mathrm{Ti}^{+4}$ aumentam no mesmo sentido. Nos elementos traços os teores de $\mathrm{Sr}$ das amostras de numero 5726 e 5716 são muito altos. O contrário acontece na amostra 5725. Interessante também é o aumento do $\mathrm{Rb}^{+}$da rocha 1 para 4 . Isto é correlacionado com o aumento do $\mathrm{K}^{+}$devido ao aumento do teor em mica clara, como o aumento de $\mathrm{P}^{+5}$ mostra o aumento do teor dos minerais fosfáticos na rocha do tipo 5 para 1 .

Na figura 3 é mostrada a distribuição das ETR para rocha total/condrito. Observa-se um leve aumento das ETRL (leves) e quase constantes ETRP (pesados), o que é típico para sedimentos. A pequena anomalia negativa do Eu também é típico para esta rocha.

Uma anomalia positiva de Ce pode ser um resultado de sedimentação de um protolito erodido sob condições oxidantes com dissolução dos $\mathrm{ETR}^{3+}$ e enriquecimento relativo de $\mathrm{Ce}^{4+}$ (Wedepohl 1978).

\section{DISCUSSÃO DOS RESULTADOS}

Neste capítulo serão discutidas, além das implicações regionais e da posição estratigráfica, a origem e a composição do protolito, as texturas e a composição dos vários minerais, como também as limitações impostas para a composição mineral em relação à composição da rocha-mãe.

\begin{tabular}{|c|c|c|c|c|c|}
\hline Tipo & $\mathbf{1}$ & $\mathbf{2}$ & $\mathbf{3}$ & $\mathbf{4}$ & $\mathbf{5}$ \\
\hline Num. am. & $\mathbf{5 7 2 6}$ & $\mathbf{5 7 1 1}$ & $\mathbf{5 7 1 7}$ & $\mathbf{5 7 1 6}$ & $\mathbf{5 7 2 5}$ \\
\hline $\mathrm{SiO}_{2}$ & 62.28 & 74.16 & 64.80 & 69.11 & 76.10 \\
\hline $\mathrm{TiO}_{2}$ & 0.07 & 0.15 & 0.10 & 0.34 & 0.31 \\
\hline $\mathrm{Al}_{2} \mathrm{O}_{3}$ & 16.99 & 13.23 & 21.20 & 17.31 & 17.70 \\
\hline $\mathrm{Fe}_{2} \mathrm{O}_{3}{ }^{*}$ & 1.84 & 1.74 & 1.93 & 2.81 & 2.38 \\
\hline $\mathrm{MgO}$ & 2.41 & 0.77 & 0.52 & 0.27 & $<0.05$ \\
\hline $\mathrm{CaO}$ & 0.19 & -- & 0.02 & -- & $<0.02$ \\
\hline $\mathrm{Na}_{2} \mathrm{O}$ & 0.14 & 0.05 & 0.14 & 0.09 & -- \\
\hline $\mathrm{K}_{2} \mathrm{O}$ & 1.79 & 1.90 & 5.69 & 4.59 & 1.13 \\
\hline $\mathrm{P}_{2} \mathrm{O}_{5}$ & 11.68 & 4.71 & 2.23 & 1.35 & 0.04 \\
\hline $\mathrm{S}$ & -- & 0.01 & 0.02 & 0.14 & 0.1 \\
\hline $\mathrm{B}[\mathrm{ppm}]$ & -- & -- & 473 & -- & 19 \\
\hline $\mathrm{F}$ & 0.23 & 0.26 & -- & 0.23 & -- \\
\hline $\mathrm{P} . \mathrm{F}$. & 3.5 & 1.8 & 2.7 & 2.8 & 0.70 \\
\hline $\mathrm{Total}$ & 101.6 & 98.78 & 99.35 & 99.04 & $<98.57$ \\
\hline $\mathrm{Ba}$ & 461 & 62 & 160 & 163 & 64 \\
\hline $\mathrm{Co}$ & 32 & 16 & 13 & 12 & $<8$ \\
\hline $\mathrm{Cr}$ & 8 & 16 & 16 & 45 & $<8$ \\
\hline $\mathrm{Cu}$ & 15 & 8 & 12 & 8 & 13 \\
\hline $\mathrm{Nb}$ & -- & 17 & 12 & 30 & 27 \\
\hline $\mathrm{Pb}$ & 50 & 56 & $<20$ & 58 & $<20$ \\
\hline $\mathrm{Rb}$ & 52 & 43 & 161 & 113 & 40 \\
\hline $\mathrm{Sr}$ & 1646 & 1132 & 364 & 4296 & 75 \\
\hline $\mathrm{V}$ & 11 & -- & $<8$ & 23 & 30 \\
\hline $\mathrm{Y}$ & 75 & 37 & 37 & 47 & 21 \\
\hline $\mathrm{Zr}$ & 51 & 103 & 20 & 189 & 218 \\
\hline $\mathrm{MgO} / \mathrm{FeO}$ & 1.45 & 0.49 & 0.43 & 0.11 & $<0.02$ \\
\hline
\end{tabular}

Tabela 2: Comparação química dos diferentes tipos de rochas 1 a 5 . $\mathrm{Fe}^{2} \mathrm{O}^{3 *}=$ ferro total calculado como $\mathrm{Fe}^{3+} ; \mathrm{FeO}^{*}=$ ferro total calculado como $\mathrm{Fe}^{2+}$

Table 2: Chemical comparison of the different roch types 1 to $5 . \mathrm{Fe}^{2} \mathrm{O}^{3} *=$ total iron calculated as $\mathrm{Fe}^{+++} . \mathrm{FeO}{ }^{*}=$ total iron calculated as $\mathrm{Fe}^{++}$.

\section{Posição estratigráfica}

Através de observações de campo e estudos bibliográficos conclui-se que a deposição do protolito rico em alumínio, boro e fósforo ocorreu durante o estágio inicial do Rift Espinhaço (1750Ma; Almeida Abreu 1993). Tal deposição teve lugar provavelmente na superfície do relevo existente da Formação Barão de Guacuí, a qual sofreu posteriormente uma transformação superficial em paleossolo (Knauer \& Schrank 1994).

Os contatos observados entre as formações Barão de Guacuí, Bandeirinha e São João de Chapada sempre foram interpretados como sendo por discordância angular e/ou erosiva. Knauer (1989) e Knauer \& Schrank (1994) descrevem contatos gradativos entre 


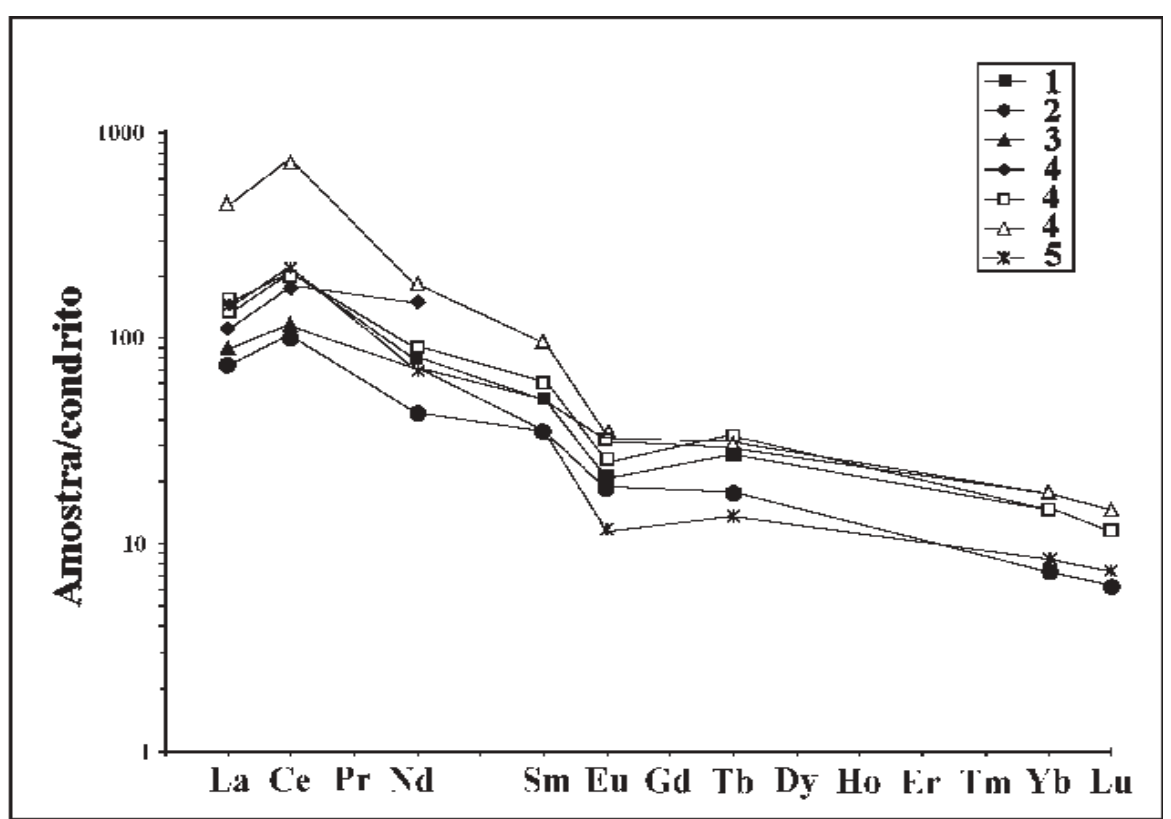

Figura 3: Distribuição do padrão de ETR normalizada com relação ao condrito para as rochas que contêm lazulita.

Figure3: Rare earth element distribution of the lazurite bearing rocks. Normalized against chondrite.

as formações Barão de Guacuí e Bandeirinha. Não é possível a interpretação correta deste contato, pois ele é geralmente paralelizado e conseqüentemente mascarado pelo falhamento posterior (figura 2).

A transformação do protolito da lazulita e outros deve ter ocorrido com a tectonização do conjunto de rochas durante e/ou após o fechamento do rift (após 1500Ma e entre 700-800Ma; Almeida Abreu 1994).

As condições desta transformação não são exatamente definidas, mas supõe-se que chegaram a condições de fácies xisto-verde (Almeida Abreu 1994).

\section{Protolito}

A composição química das rochas que contêm lazulita é caracterizada pelos altos teores em $\mathrm{Al}^{+++}, \mathrm{P}^{+5}$, $\mathrm{F}^{-}$e $\mathrm{B}^{+3}$ e teores baixos a muito baixos em $\mathrm{Na}$.

A idade dos quartzitos e quartzo-xistos é arqueano superior e/ou proterozóico inferior (Schöll \& Fogaça 1979, 1981; Almeida Abreu 1994; Dussin 1994; Silva 1995). Dentro deste contexto precisa-se levar em conta também um modelo de formação não atualístico das condições de depósito.

A evidência geológica e o comportamento dos fosfatos favorece uma deposição do protolito rico em $\mathrm{P}^{+5}$ nas partes basais da Formação Bandeirinha, nos estágios iniciais da abertura do Rift Espinhaço durante a primeira ingressão do mar (figura 4).

Uma primeira hipótese considera estes primeiros mares como provavelmente mares ou lagoas sódicas ricas em $\mathrm{Si}^{+4}$ e $\mathrm{Fe}^{++}$mas relativamente pobre em $\mathrm{Ca}^{++} \mathrm{e}$ $\mathrm{Mg}^{++}$e em geral sem $\mathrm{SO}^{4--}$ devido a falta de oxigênio (Einsele 1992). Mas não pode ser excluido, devida a presença de primeiros estromatólitos e o início da fotossíntese durante o arqueano superior, a formação local de vida orgânica, e como conseqüência, a deposição de séries contendo $\mathrm{P}^{+5}$. $\mathrm{O}$ ambiente deposicional pode ter sido subtidal, intertidal e/ou supratidal (Sond \& Gao 1985; Einsele 1992). Em ambientes costeiros, com formação de Rift, o desenvolvimento de deposição clástica, essencialmente de grãos de quartzo intercaladas com camadas conglomeráticas é óbvio, especialmente por deposições de fluxos subaquáticos como turbiditos. Estes fluxos de água fresca podem dissolver grandes quantidades de sais dos sedimentos tais como halita e/ou soda, diminuindo o conteúdo de $\mathrm{Na}^{+}$, mas deixando in situ os sais insolúveis de fósforo e de boro e pequenas quantidades de minerais de $\mathrm{Na}^{+}$e $\mathrm{Li}^{+}$necessários para a formação de dumortierita e ambligonita durante o metamorfismo posterior. Um processo semelhante ao descrito poderá ter sido responsável pelos teores de $\mathrm{Na}^{+}$ muito baixos nos sedimentos com lazulita. Por outro lado, é difícil de explicar como mares e/ou lagoas arqueanas são ricos em $\mathrm{Na}^{+}$.

O segundo tipo de ambiente que deve ser discutido é aquele desenvolvido sob condições climáticas quase que semi-áridas (Einsele 1992), pois nestes ambientes pode haver enriquecimento em elementos tais como $\mathrm{K}^{+}, \mathrm{P}^{+5}, \mathrm{~B}^{+3}$ e $\mathrm{Li}^{+}$. Debris flows que depositaram camadas de clastos grosseiros, dissolveram sais, como halita e soda, e assim contribuiram para a redução dos altos teores destes sais existentes anteriormente.

Uma terceira possibilidade foi discutida por Schreyer (1987) e compreende a alteração sericítica de rochas ácidas vulcânicas. Durante uma alteração deste tipo, $\mathrm{Ca}^{++}$e $\mathrm{Na}^{+}$serão empobrecidos seletivamente e $\mathrm{Si}^{+4}$ e $\mathrm{Al}^{+3}$ serão relativamente enriquecidos. Isto acontece ao mesmo tempo que 
ocorre a circulação de soluções hidrotermais. Elementos como fósforo, lítio e boro poderão se enriquecer e/ou se depositar em camadas de ambiente favorável.

Levando-se em consideração a área relativamente grande que os sedimentos com lazulita ocupam, o modelo costeiro e/ou lacustre sob condições semi-áridas parece ser a mais provável das alternativas.

\section{Estabilidade dos fosfatos de alumínio}

Com base nas observações das lâminas delgadas, parece que a lazulita começou crescer precocemente na história deformacional destas rochas e continuou como mineral "refratário" durante todo o processo

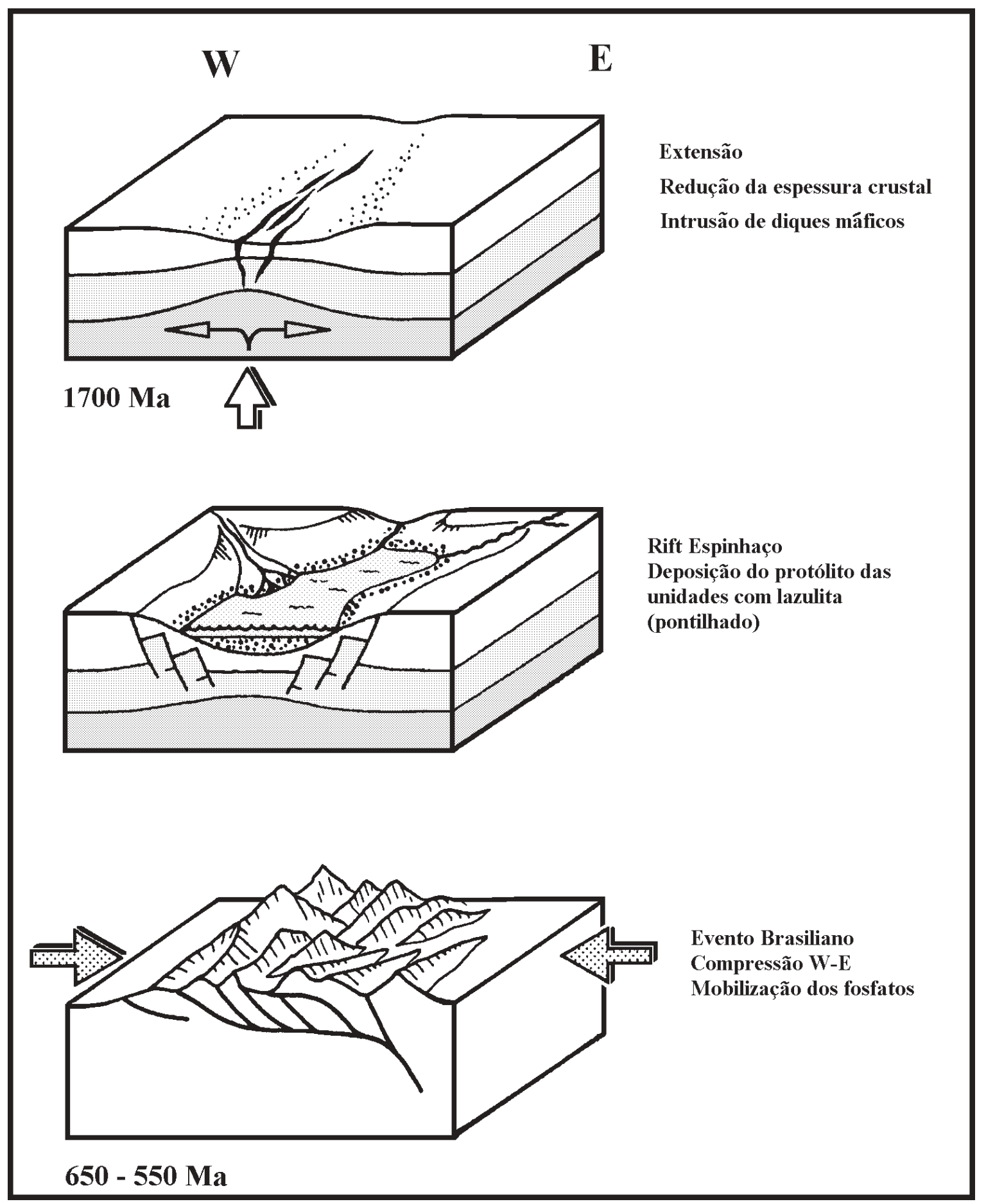

Figura 4: Seqüência esquemática dos estágios fundamentais da formação do Rift Espinhaço, sendo indicado o local da deposição do protolito das rochas com Al-P e Al-B minerais (segundo Dussin 1994; modificado).

Figure 4: Schematic sequence of the fundamental stages of Espinhaço Rift evolution, with the indication of the place of deposition of the protolite of the Al-P and Al-B minerals bearing rocks (after Dussin 1994; modified). 
metamórfico. Isto é mostrado pela aparência fresca dos blastos de lazulita e pelo aumento de tamanho dos minerais opacos fora destes blastos. As estruturas sigmoidais das inclusões demonstram que a cristalização durou até os últimos estágios da deformação. Isto foi também observado por Morteani \& Ackermann (1996). Mas parece que os compostos da lazulita continuaram a ser móveis durante um período posterior aos vários estágios tectonometamórficos que as rochas sofreram.

Grandes cristais anedrais de lazulita azul-escuro até $5 \mathrm{~cm}$, são citados na literatura (alguns, até vários $\mathrm{kg}$; Ribeiro et. al. 1995). Estes foram encontrados em veios de quartzo levemente deformados, que cortam discordantemente todas as xistosidades. Nestes veios existe também ambligonita. As condições de estabilidade da lazulita precisam ser ainda definidas.

$\mathrm{O}$ fato de que simplectitos de muscovita com lazulita muitas vezes estão envolvidos por rims de augelita, sustenta a idéia de um break-down da lazulita para augelita.

\section{CONCLUSÕES}

Investigações mais detalhadas, quer no campo, quer no laboratório, são extremamente necessárias para se obter mais dados sobre o comportamento e o campo de estabilidade das várias fases de fosfatos e de dumortierita.

Pode-se concluir que os fosfatos e boratos de alumínio foram depositados juntamente com outros sais durante um estágio relativamente precoce do Evento Espinhaço. Posteriormente, tais sais foram parcialmente dissolvidos e o restante transformado por metamorfismo.

Da estrutura interna da lazulita pode-se concluir que ela se formou durante os primeiros estágios da história metamórfica, principalmente durante um metamorfismo estático. Escassas estruturas sigmoidais internas documentam que ocorreu muito raramente um crescimento sintectônico. A estrutura interna, mostrada pelas inclusões, limita-se muitas vezes ao núcleo, enquanto as margens estão quase livre de inclusões. Em alguns casos observa-se zoneamento colorido o qual começa com azul claro no centro e um azul-escuro nas margens.

Observou-se uma mobilização da lazulita através dos planos de foliação e nas fraturas preenchidas por quartzo que cortam camadas superiores e inferiores, e também uma transformação tardia que alterou as bordas de lazulita em augelita. Infelizmente, não existem ainda dados confiáveis sobre as condições P-T das rochas da Formação Barão de Guacuí e/ou da Formação Bandeirinha.

Para o contato do Supergrupo Rio Paraúna com o Supergrupo Espinhaço pode-se pensar que uma diferença textural e granulométrica entre as rochas da Formação Barão de Guacuí típica e as camadas superiores (cerca de até $1 \mathrm{~m}$ ) próximas de uma zona de cisalhamento intensivo, associada a um cavalgamento, podem influenciar a interpretação deste contato (figura
2).

Parece ser possível que os protolitos da lazulita, dumortierita e turmalina foram depositados sobre uma base já consolidada de mica-quartzo-xisto e posteriormente ambos foram foliados. A existência de geralmente duas foliações na Formação Barão de Guacuí e somente uma foliação diretamente abaixo do contato com o Supergrupo Espinhaço, parece claramente sustentar este hipótese.

\section{AGRADECIMENTOS}

Agradecemos pelas informações úteis aos $\mathrm{S}^{\text {rs. }}$ geólogos Dr. J.P. Cassedanne (Rio de Janeiro), A. C. C. Fogaça, Dr. P. A. Almeida Abreu e $M^{\text {sc. }}$ G. Knauer (Belo Horizonte).

A Sra. Mader (Kiel) que executou as análises de microssonda.

Ao Centro de Geologia Eschwege em Diamantina e o seu diretor Dr. P.A. Almeida Abreu que nos prestaram suporte logístico para o trabalho de campo.

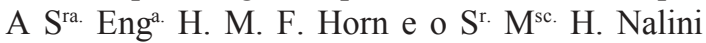
(Belo Horizonte) que nos ajudaram na revisão final do texto.

\section{REFERÊNCIAS BIBLIOGRÁFICAS}

Almeida Abreu, P.B. 1989. Geologia das Quadrículas Onça e Cuiabá (Gouveia-MG) - região mediana central da Serra do Espinhaço Meridional. $\mathrm{M}^{\text {sc. }}$ Thesis, Inst. Geociênc. UFRJ: 75p.

Almeida Abreu, P. B., Pflug, R. \& Schorscher, H. D. 1992. Cover/ Basement relationships in the southern Serra do Espinhaço, Minas Gerais, Brazil. Zbl. Geol. Paläont. Teil I: 1749-1760.

Almeida Abreu, P.B. 1993. A evolução geoquímica da Serra do Espinhaço Meridional, Minas Gerais, Brasil. Albert-LudwigsUniversität Freiburg, Dissertation: 150p.

Bank, H. 1972. Blauer und dunkelgrüner duchsichtiger Lazulith aus Brasilien - Zt. Dtsch. Gemmol. Ges., 21:219-221.

Cassedanne, J.P. \& Cassedanne, J.O. 1975. Note sur la lazulite de la region de Diamantina (MG).- An. Acad. Bras. Cienc., 47: 283 288.

Cassedanne, J.P. 1990. Un nouveau materiaux gemme: le quartzite a lazulite (Bahia-Brasil).- Revue de Gemmologie, 105: 17 - 18.

Cassedanne, J.P., Cassedanne, J.O. \& Carvalho, H.F. 1989. Origine des lazulites liées a des accidents ferriféres dans des quartzites a dumortierite (Serra da Vereda, Bahia, Bresil).- An. Acad. Bras. Cienc., 61: 59 - 72.

Dorr, J.V.N. 1969. Physiographic, stratigraphic and structural development of the Quadrilátero Ferrífero, Minas Gerais, Brazil. US Geolog. Surv. Prof. Pap. 641-A: 110p.

Dussin, I.A. 1994. Evolution structurale de la region de l'Espinhaço Meridional, Bordure Sud-Est du Craton São Francisco, Brésil. Université d'Orléans, These de docteur: 260p.

Eberle, W. 1970. Petrographische und geologische Untersuchungen in der Gegend von Diamantina (Espinhaço Zone, Minas Gerais, Brasilien). Ruprecht-Karl Universität, Heidelberg, Dissertation, Teil 1: 45p.

Eberle, W. 1972. Petrographische und geologische Untersuchungen in der Umgebung von Diamantina (Espinhaço-Zone, Minas Gerais, Brasilien).- Beih. Geol. Jb., 121: 5-58.

Einsele, G. 1992. Sedimentary basins: Evolution, facies and sediment budget. Springer Verlag, Berlin, 628p.

Fogaça, A.A.C. \& Schöll, W. U. 1984. Estratigrafia e tectônica das rochas Arqueanas e Proterozóicas da região de Guinda e Gouveia (MG).- XXXIII Congr. Bras. Geol., Anais...: 2.639-2.653.

Herrgesell, G. \& Pflug, R. 1986. The thrust belt of the southern Serra do Espinhaço, Minas Gerais, Brazil.- Zbl. Geol. Paläont. 1985. Teil I: $1405-1414$ 
Hoffmann, C,. 1979. Augelite, a rare aluminium phosphate: first find in a metamorphic environment.- N. Jb. Miner., Abh., 136: $1-9$.

Knauer, L.G.1990. Evolução geológica do Pré-Cambriano da porção centro-leste da Serra do Espinhaço Meridional e metalogênese associada. UNICAMP. Campinas, Diss. de Mestado: 298p.

Knauer, L.G. \& Schrank, , A. 1994. A origem dos filitos hematíticos da Serra do Espinhaço, Minas Gerais. Geonomos, V1/1: 33$38 \mathrm{p}$.

Machado, N., Schrank, A. Abreu, F.R, de, Knauer, L.G. \& Almeida Abreu, P.A. 1989. Resultados preliminares da geocronologia $\mathrm{U} / \mathrm{Pb}$ na Serra do Espinhaço Meridional. SBG/Núcleo MG, Bol. 10: $171-174$.

Morteani, G. \& Ackermand, D. 1996. Aluminium Phosphates in muscovite kyanite metaquartzites from Passo di Vizza (Alto Adige, NE Italy).-Europ. Journ. Miner, in press.

Pecora, W.T. \& Fahey, J.J. 1950. The lazulite-scorzalite isomorphous series. Am. Mineral., 35: 1-18.

Pflug, R. \& Carvalho, R.T. 1964. A evolução estrutural da região de Gouveia, Serra do Espinhaço, Minas Gerais. DNPM, Div. Geol. Min. 213: 37p.

Pflug, R. 1965. A geologia da parte meridional da Serra do Espinhaço e zonas adjacentes, Minas Gerais. DNPM Div. Geol. Min. Bol. 226: 55p.

Pflug, R. 1968. Observações sobre a estratigrafia da Série Minas na região de Diamantina, Minas Gerais. DNPM Div. Geol. Min.,
Notas Prelim. Estud. 142: 20p.

Pflug, R., Hoppe, A. \& Brichta, A. 1980. Paleogeografia do Précambriano na Serra do Espinhaço, Minas Gerais, Brasil. Nuevos Result. Pesqu. Alemanha Latinoam., Boppard DFG: 33-43.

Ribeiro, S.H., Karfunkel, J., Chaves, M.S. \& Banko, A. 1995. Gemíferos da Cordilheira do Espinhaço. $8^{\circ}$ Simp. Geol. Minas Gerais, SBG/Nucleo MG, Diamantina, Anais...: 39-40.

Silva, R.R. 1995. Contribution to the stratigraphy and paleogeography of the Lower Espinhaço Supergroup (Mesoproterozoic), between Diamantina and Gouveia, Minas Gerais, Brazil. Universität Freiburg, Dissertation: 115p.

Schöll, W.U. \& Fogaca, A.C.C. 1979. Estratigrafia da Serra do Espinhaço na região de Diamantina (M.G.). 1 Simp. Geol. Minas Gerais, Diamantina, Anais...: 55 - 73.

Schöll, W.U. \& Fogaça, A.C.C. 1981. Mapeamento geológico das quadrículas Guinda e Gouveia (MG). - Projeto Mapeamento Espinhaço - DNPM/CPRM, Belo Horizonte: 51p (inédito).

Schreyer, W. 1987. Pre- or synmetamorphic metasomatism in peraluminous metamorphic rocks?- in: Helgeson, H.C. (ed.): Chemical transport in metasomatic processes, Springer Verlag, Heidelberg: 265-296.

Song, T. and Gao, J. 1985. Tidal sedimentary structures from the Upper Precambrian rocks of the Ming tombs district, Beijing (Peking), China.- Precambrian Research, 29: 93-107.

Wedepohl, K.H. 1978. Handbook of geochemistry. Springer Verlag. Berlin, Heidelberg, New York.

Adolf Heinrich Horn: e-mail headhorn@general.igc.ufmg.br

Giulio Morteani: e-mail morteani@mineral.min.chemie.tu-muenchen.de Dietrich Ackermand: e-mail nmp55@rz.uni-kiel.d400.de 